\title{
What echolocation does and doesn't mean for language
}

Brett Reynolds

Humber College, Toronto, Canada

brett.reynolds@humber.ca

https://orcid.org/0000-0003-0073-7195

\begin{abstract}
There is an argument from analogy that goes like this: echolocation is a cognitive specialization in bats and language is similarly a cognitive specialization in humans; consequently, because echolocation is a trait that is the result of natural selection, the language faculty must also be. I show that echolocation is a latent ability in humans which cannot have been selected for, i.e., it is a spandrel. This turns the tables on the argument from analogy, showing that either the analogy was false, or that the correct conclusion is that the language faculty is likely a spandrel. I then argue that natural selection of a propensity to notice and attend to the relevant input is likely to have led to the language faculty.
\end{abstract}

Keywords: language, evolution, innateness, echolocation, human, bat, noticing

\subsection{Introduction}

The prevalent view in linguistics is that humans have an innate language faculty, absent in all other animals. When linguists speak of this language facility, they are usually discussing a facility in processing language. This is at least in part because

the development of the Turing machine, in conjunction with parallel work by John von Neumann on computer architectures and Claude Shannon in information theory, occurred at the same time as the death of behaviourism and the arrival of cognitivism in psychology. Indeed, young leaders in psychology and linguistics like George Miller and Noam Chomsky were strongly influenced by these developments in computational theory. In part because of these historical roots, the 
notion of computation is central to the project of modern cognitive science and the framework of cognitivism. (Chater et al. 2015:13)

If this computational focus is in any doubt, one need only consider discussions of recursion (Hauser, Chomsky \& Fitch 2002), the Chomsky hierarchy (Jäger \& Rogers 2012), and the poverty of the stimulus argument (Pullum \& Scholz 2002). This focus on processing stems from the perceived difficulty of language learning, something that has been seen as near impossible without some innate knowledge of structures (e.g., Baker 1979). Thus, many feel that without a specialized processor, we, like apes, would be entirely unable to learn language.

Clearly, something distinguishes our ubiquitous success at learning language and apes' complete failure (Hauser et al. 2014). And given that humans begin to learn languages as infants in every culture, it's reasonable to assume that this difference is innate. Many argue that that distinguishing feature is a language faculty. One minor argument in favour of this positions is the following argument from analogy: echolocation was selected for in bats and since language is similar to echolocation in important ways, it is reasonable to conclude that a language faculty also evolved as a result of natural selection. I take this argument apart and show why comparisons to echolocation, if apt, actually show that our language capacity is a spandrel in the sense of Gould and Lewontin (1979).

This leaves open the question of what distinguishes our language ability from that of other primates, who lack any obvious language ability. I suggest a possibility that appears to have received little attention: humans have an innate propensity to notice and attend to the relevant input. Such a noticing instinct can explain abilities that are considered to be analogous to language.

In the remainder of $\S 1$, I consider echolocation, its similarities to language, the argument from analogy, and the latent human capacity for echolocation. In $\S 2$, I set out the case for the complexity of echolocation and language, a factor that is key to the argument from analogy. In $\S 3, \mathrm{I}$ argue that the fact that echolocation is a latent human ability means that it must be a spandrel in humans, but that it is indeed the result of natural selection in bats. In $\S 4$, I consider the argument from analogy under this understanding of echolocation as a spandrel. In $\S 5$ I argue that the evidence for 
geneticization of a language faculty is weak while there is much more reason to believe that and noticing certain types of input has a genetic component.

\subsection{The case of echolocation}

Parallels have often been drawn between the evolution of echolocation in bats and the language facility in humans (e.g., Fitch, Hauser \& Chomsky 2005; Jackendoff 2002:79; Putnam 1992). Rarely are the similarities set out in any significant detail at all. Fitch, for instance, says merely that "chimpanzees do not have language or music, any more than humans have echolocation" (2014:346). Pinker and Bloom assert that "the ability to use a natural language belongs more to the study of human biology than human culture; it is a topic like echolocation in bats or stereopsis in monkeys, not like writing or the wheel" (Pinker \& Bloom 1990). Jackendoff explains that "the central claim of generative grammar is that the language faculty is a cognitive specialization, and we claim that cognitive specializations in every other species are the consequence of natural selection" (Jackendoff 2002:427), and cites echolocation as an example of a cognitive specialization. He suggests that echolocation cannot be just attributed to "“some additional feedback loops' or the "freeing up of some resources"” (Jackendoff 2002:79) and similarly assumes, "along with Pinker and Bloom (1990), ... that the complexity and specialization of language precludes it being simply a natural development from (or spandrel of) increased memory, planning abilities, motor functions, or other more general functions." Tooby, Cosmides, and Barrett (2005), argue for "circuits for ... echolocation" (308:f) and language as something produced by natural selection, not as a by-product of, for instance, visual processing capabilities and binaural hearing. My contention is the contrary: that that is exactly what echolocation and language are.

The following seem to be undisputed points of comparison between language and echolocation: (1) The involvement of audition, (2) vocalization, (3) complex cognitive processing, and (4) a typical developmental pattern in which the ability appears at a very young age in the respective species.1 More tendentious are the claims that both abilities are:

1 In the little brown bat, for instance, landing buzzes, vocalizations used to assess the landing area, are used even in the first flights, around the age of 19-20 days (Buchler 1980). 
1. Functional, having a particular purpose or purposes;

2. Adaptive, conferring increased reproductive success;

3. Complex, not the kind of thing that could happen without many significant changes;

4. Genetically or epigenetically determined.

While agreeing with Vernes (2017) that bats and echolocation are potentially fruitful sources of insight into human language, I will argue that the comparisons that are typically made between human language and bat echolocation are misleading on at least one of two main counts. First, it may be the case that echolocation and language simply are not sufficiently similar in the ways the writers intend. Evans and Levinson (Evans \& Levinson 2009), for instance, argue that "The biological underpinnings for language are so recently evolved that they cannot be remotely compared, for example, to echolocation in bats" (446). If they are right, then the above-mentioned arguments by analogy are fallacious, and the points I make below are merely minor factual corrections that do not change the fallacy. On the other hand, it may be the case that the two skills are indeed comparable, but the nature of the similarity is not as the writers believe it is. This is the argument I will develop here. If true, this would admittedly not be a major blow to the arguments that the language faculty is a cognitive specialization and the consequence of natural selection, but it would undermine certain arguments along those lines.

\subsection{Echolocation as a general latent human ability}

The major factual error in all the comparisons is the claim that bats have echolocation and humans don't. Humans do indeed have the capacity for echolocation, a fact that has been known since at least the 1940s, when the word echolocation was coined (Griffin 1944). The vast majority of us simply never learn to echolocate, in the way that many of us never learn to wiggle our ears despite having auricular muscles that could accomplish this (Bérzin \& Fortinguerra 1993). Not only do humans have the capacity for echolocation, but blind people who have learned to use it seem to be comparable in ability to bats in certain respects. In one study, "experts were able to discriminate horizontal offsets of stimuli as small as $\sim 1.2^{\circ}$ auditory angle in the frontomedial plane, a resolution approaching the maximum measured precision of human spatial hearing and comparable to that found in bats performing similar tasks" 
(Teng, Puri \& Whitney 2012:483). It would clearly be foolish to claim that human echo-locative capabilities are equal to those of bats in all respects, but this should be sufficient establish that the human echolocation capabilities are remarkably complex (Dodsworth, Norman \& Thaler 2020) and that comparisons to bat abilities are not unreasonable.

It is important to note that even though these abilities are present in humans, they are most likely not employed by the vast majority of people (see Stroffregen \& Pittenger 1995 for a contrary view) and that many who use sound to echolocate appear to use environmental sounds rather than intentionally producing the taps or clicks that expert echolocating humans - along with various bat species (Yovel, Geva-Sagiv \& Ulanovsky 2011) - employ. Even among blind people, usage rates appear to be only about 20-30\% (Thaler 2013), though this is likely underreported due to unconscious use.2 Teng and Whitney provide evidence that "the crucial spatial resolution component of the skill, while not immediately accessible to most untrained subjects, can be readily learned" (Teng \& Whitney 2011:6; emphasis added). In other studies, blind participants - not necessarily expert echolocators - were significantly better than sighted participants at identifying the location and characteristics of objects by listening alone, though they "had difficulty in describing physical features of the object" (McGrath, Waldmann \& Fernström 1999:8). In addition, blind participants were able to detect objects at a greater distance than sighted participants (Schenkman \& Nilsson 2010). Evidence for significant use among sighted people not specifically trained in echolocation appears to be lacking, but with suitable training or experience, echolocation is readily learned (Dodsworth, Norman \& Thaler 2020; Kolarik et al. 2014).

Clearly, then, humans have the ability to process the spatial information contained in echoes, and some eventually learn to do so with high levels of effectiveness. As far as we know, however, unlike bats, hearing infants do not typically begin using this information to learn about their surroundings. Nor do they appear to instinctively produce sounds analogous to the screams, buzzes, and clicks that bats employ for

2 Unconscious use "was evidenced early on by erroneous self-reports of blind individuals that their ability to avoid collision was based on sensitivity to air pressure changes on the face" (Rosenblum, Gordon \& Jarquin 2000:202). 
echolocation. Nevertheless, children who were born blind are often able to avoid obstacles without any training (Kolarik et al. 2014), and there is anecdotal evidence that they instinctively do vocalize for the purposes of echolocation.

\subsection{The case for complexity}

The argument from analogy relies on the claim that both echolocation and language are highly complex traits. This does not appear to be in dispute. Nevertheless, I will briefly attempt to build a case to support it.

As I said, expert opinion supports the idea that these are complex skills. Language learning is seen to be so complex that it could not be learned without some innate knowledge (Baker 1979). Countless experts have described it as a complex system (e.g., Beckner et al. 2009). In contrast, the complexity of bat echolocation isn't as much of a topic of academic interest. Nevertheless, numerous experts describe it as complex (Wilkinson 2003; Weinbeer \& Kalko 2007).

A more objective approach is to think that something that is complex has many parts and takes a lot of language to describe it. Under this understanding, any human language would be complex. An example would be the Cambridge grammar of the English language (Huddleston \& Pullum 2002), which requires over 1,750 pages just to do a superficial overview of the morphology and syntax of spoken and written Standard English. Echolocation seems likely to be much easier to describe. The basics of sending out a sound and calculating where something is based on the timing of the echo as it arrives at the bats' ears can be expressed with a few lines of math.

Disentangling this sound from the sound of other bats and environmental sounds, adds complexity, and deciding whether the object is an insect to be eaten or an object to be avoided makes it much more complex.

The problem seems much easier than describing language, but it would be far from a trivial undertaking. An example would be quantitative analyses of the echolocation strategies of bats (Aihara, Fujioka \& Hiryu 2013). This is orders of magnitude shorter than a description of English but still quite long and far from complete.

In sum, then, it seems that language is the more complex of the two but that both are indeed complex cognitive skills. The fact that these are complex cognitive skills, it is 
argued, means that they must have arisen through natural selection, not as by-products or spandrels.

\subsection{The evolution of echolocation in humans: Adaptation, exaptation, or spandrel}

Setting aside, for the moment, echolocation in bats, I'd like to consider the situation in humans. If our capacity for echolocation is latent, then, crucially, it could confer no reproductive advantage and could not be subject to natural selection in humans. It has no role to fit, so it can be neither ADAPTATION (designed to fit its current role) nor EXAPTATION “fit (aptus) by reason of (ex) their form" (Gould \& Vrba 1982:6). The capacity for echolocation in humans seems to be a paradigm case of a SPANDREL, a capacity that has not been selected for but comes into being as a necessary by-product of other capacities that have (Gould \& Lewontin 1979). In particular, given that "processing of click-echoes recruits brain regions typically devoted to vision rather than audition in both early and late blind echolocation experts" (Thaler, Arnott \& Goodale 2011:1), human echolocation may crudely be said to span hearing and visual processing.

\subsection{Echolocation in bats}

The situation is different in echolocating bats. There, we find overwhelming evidence of use conferring a survival advantage, a role, which constitutes a mechanism for natural selection. Non-echolocating bats have a much slower recovery rate after an initial sound than echolocating bats, which "suggests that fast temporal resolution is perhaps the most important of the necessary neural adaptations for echolocation" (Grinnell \& Hagiwara 1972:83). The underlying cognitive abilities are also commonly, though not always, accompanied by physical attributes such as relatively large outer ears, acoustic fovea in the inner ear (Schuller \& Pollak 1979), and the ability to produce loud ultrasound vocalizations, which in itself seems to be partially a by-product of large flight muscles (Speakman 1993).3 All of these constitute evidence of evolution through natural selection. But the process that set this process in motion also need to be considered. 
As in humans, bats likely had a latent echolocation ability, which, if true, would make this an exaptation (Gould \& Vrba 1982), at least initially. One reason to believe so is that "the typical high-frequency sensitivity of small non-echolocating mammals would have been sufficient to support initial echolocation in the early evolution of bats" (Heffner, Koay \& Heffner 2006:17). Moreover, a variety of distantly related species, such as shrews (Gould, Negus \& Novick 1964), also echolocate using ultrasound, though we do not find it widely used in many intermediate taxa. Even in species that do not typically employ echolocation, there is evidence for a latent ability; for instance, blinded rats can use sound to navigate (Rosenzweig, Riley \& Krech 1955) although it's unclear whether sighted rats do so.

Another element is the production of ultrasound vocalizations in apparently-nonecholocating rodents (Roberts 1979), which appears to be for purposes of environment characterization, perhaps "comparable to human hearing whether one calls into a beech forest or into a reverberant wine cellar" (Siemers et al. 2009:595). In other words, much of the physical and cognitive apparatus is present is related species and would have been available for recruitment by the predecessors of echolocating bats (see Speakman 1993 for an overview of evolutionary forces leading to selection of echolocation).

\subsection{The case for selection for language}

To reiterate, it could be argued that comparisons between language and echolocation are inappropriate. But if we take them seriously, then we should also take seriously the possibility that language, too, is a spandrel. This idea is diametrically opposed to the position set forth by many of those wielding the comparison. Pinker and Bloom argue that "complex design to carry out some reproductively significant function" should be "clear criteria for when selectionist ... accounts should be invoked" (Pinker $\&$ Bloom 1990). Echolocation in bats meets both these criteria (complexity and reproductive significance), but echolocation in humans only meets the criterion of complexity.

On the other hand, Pinker and Jackendoff call it "inconceivable that a system for speech perception in humans could have begun de novo" (2005:206). Yet, if relatively complex echolocation is a latent ability in a wide variety of animals, and therefore available without being selected for, then it seems plausible that much of the 
cognitive machinery needed to deal with complex language too, may have existed before it was used for language. As in bats, fast temporal resolution would have had to evolve (Liberman et al. 1967), and memory would likely have improved (Hurford 2012:chap. 6). Nevertheless, there is nothing in this argument from analogy that precludes a latent language capacity for language in pre-linguistic humans in the way that we have the latent capacity for echolocation.

Despite looking hard, though, we have not found unambiguous evidence for similar latent linguistic capacity in other animals. Nevertheless, there is suggestive evidence that proto-language capacities are latent in African grey parrots, for example (Pepperberg 1983). Clearly, then, it is not an inevitable conclusion that language capacity is a spandrel, but if the arguments by analogy are rerun under this latent human echolocation observation, then they point in this direction.

\subsection{The case for genetecism}

The comparisons are not used simply to bolster the case for selection (and thus nativism) but are also used to argue more specifically for genetecism. For instance, Culicover and Jackendoff write, "if Narrow UG is innate, it must be coded genetically, just like any specialized cognitive capacity in any animal, such as bat sonar" (Culicover \& Jackendoff 2005:13). There is a level at which this is true, at which almost anything involving an organism can be said to be genetic: susceptibility to poisoning by curare and stubbing a toe, for example. The question is whether it is reasonable and useful to call such things genetically encoded. When we're talking about something like lactase persistence where a single allele fully correlates with the trait in many global populations (Enattah et al. 2008:57), it's hard to see it any other way. But with an ability like echolocation in humans, which is merely a potential, a side effect of various other abilities that are coded by the interaction of a wide variety of genes, some of which may do things like expand areas of the brain that are used a lot, this is harder to maintain. We could argue that evolution of language abilities proper and geneticization occurred after the basic foundations for language were in place. As Putnam (1992) points out, though, similar arguments could be made for our linguistic abilities being the result of the evolution of a more powerful general intelligence. 
When Putnam tries to get around this point and show that geneticization of a language faculty proper must have occurred, he runs into serious logical and factual problems. He claims that "examination of the bat's brain shows that there has been a tremendous enlargement of the centers connected with hearing (they fill about seven-eighths of the bat's brain), as compared to other mammals (including, presumably, those in its evolutionary past). Clearly, a lot of the bat's echo-locating ability is now "innate"" (1992:405).

The uncited claim about the "centres connected with hearing" is factually inaccurate. Using brain size measurements of 27 species of aerial insectivore bat (Hutcheon, Kirsch \& Garland 2002:168), I calculate that the ratio of the auditory nuclei volume to hippocampus volume ranges from $12 \%$ to $46 \%$ (median $32 \%$, mean $33 \%$ ), and ratio of the auditory nuclei volume 4 to the main olfactory bulb volume ranges from $33 \%$ to $474 \%$ (median of $104 \%$, mean of $149 \%$ ). These three areas do not come close to constituting the full volume of the brain, so it's difficult to imagine where Putnam comes up with seven eighths. In comparison, humans have a Heschl's gyrus (HG), the area associated with auditory processing, with a mean total volume of $6,473 \mathrm{~mm} 3$ (Rademacher et al. 2001:677), which compares to a mean human hippocampus volume of 3,905.74mm3 (Fortin et al. 2008:2995) and a mean total olfactory bulb volume of 106.60mm3 (Rombaux et al. 2010:1069). This puts the volume of $\mathrm{HG}$ at $166 \%$ of that of the hippocampus and $6,072 \%$ of that of the olfactory bulb, suggesting that the relative size of the auditory processing areas in bats is comparable in volume to that in humans. The fact that Putnam gets his evidence wrong doesn't necessarily mean his conclusions are also incorrect, but it does invalidate his argument.

The logical problem is that even if the centres connected with hearing were relatively large, it would necessarily follow that the enlargement is the result of something now being "innate" or geneticised. It's known that ontogenetic changes can occur in the volume of brain areas related to extensive use in cognitively demanding tasks. Taxi drivers in London, for example, who have memorized the routes of the city have enlargements in relevant brain areas (Maguire et al. 2000). So, the size of these areas

4 It appears that Putnam isn't the only one unclear about what part of the brain is being measured. AUDITORY NUCLEI is a term not widely used. I assume, perhaps incorrectly, that it refers to the auditory cortex. 
in bat brains may be no more innate than the size of muscles in body builders, the main difference being that the extensive use of echolocation is pervasive and essential to survival among bats, while bodybuilding is a minor pursuit among humans. Of course, it is possible that geneticization of certain capacities, such as improved memory and better temporal resolution, has occurred in humans, but these are external to the "language faculty" proper, and nothing in the argument by analogy supports the idea that anything internal has geneticized.

\subsection{Noticing and attention is genetic}

There is ample evidence that organisms are innately (and genetically) predisposed to notice certain types of input (Seligman 2016). A paradigmatic example of a monogenic ability involves the noticing of bitter tastes by mice. Different strains of mice, when given bitter-tasting brucine, exhibit "strong aversion or total indifference depending on presence or absence of the SOA-Tasting allele" (Whitney \& Harder 1994:1145). For many other types of input, it is not possible, currently, to associate noticing to individual alleles, and indeed it is quite likely that noticing is not monogenic. Nevertheless, we can infer a genetic component based on breeding history. Consider the difference between wild foxes, which are poor at noticing human gaze direction, and domesticated foxes, which are much better (Hare et al. 2005; notwithstanding Lord et al. 2020). Another example is Japanese monkeys, which attend to pictures of snakes faster than they do to pictures of flowers even when they have no experience with snakes at all (Shibasaki \& Kawai 2009). "The upshot of these examples is that learning itself may be quite different depending on how prepared the organism is for the particular contingency he confronts" (Seligman 2016:581).

Certainly, brain areas may develop both ontogenetically and phylogenetically to process different kinds of input. But the input must be noticed and attended to. While organisms are innately (and genetically) predisposed to attend to certain inputs (as humans to faces), other input must be brought to the organism's attention (as most humans to the spatial information in sound that allows echolocation), and still other inputs are simply impossible for particular organisms to notice without some kind of technological mediation. The vast majority of us, for example, are insensitive to light in the $440 \mathrm{~nm}$ wavelength range. Yet, it is estimated, that $12 \%$ of women have four 
classes of retinal cone cell instead of the standard three, the extra cone being sensitive to light in that range. Interestingly, the evidence suggests that only a very small number of such women notice 440nm light (Jordan et al. 2010). One of the various possible explanations for the lack of functionality is that the visual processing centres of the brain do not attend to their output. If so, then it may be the case that these women could be trained to see the new colours, analogous to the way that adults with amblyopia (Zhou et al. 2006) or presbyopia (Polat et al. 2012) can improve contrast sensitivity and visual acuity with training, or analogous to the way that people who become blind may learn to echolocate. This may, in turn, lead to increases in the associated area(s) of the brain. In each case, the training forces the participants to attend to information in the visual input that they had not previously attended to.

A lack of innate attention may also explain why apes, for instance, fail to learn human languages despite our best effort to train them: not because they can't process the signal, though perhaps they can't, but because they don't even notice it. This kind of interspecies barrier cuts both ways. Clearly, humans have the cognitive capacity to understand a wide variety of animal messages from bee waggles (von Frisch 1967) to colour-specific alarm calls in prairie dogs (Slobodchikoff, Paseka \& Verdolin 2009). Yet only a handful of humans have ever actually noticed - as opposed to being shown or told - that these messages are there to be decoded, let alone managed to decode them. It is not a lack of ability to understand such messages that holds the rest of us back; it is because we do not attend to the relevant information in the input.5 And this argument does not beg the question. We don't need language-specific innate knowledge (e.g., Merge) to notice that there may be an interesting signal in spoken or signed language any more than we need innate knowledge of bee waggles to notice them.

More evidence for the role of attention comes from kin recognition in birds. "In several gull species, parents fail to recognize their young until the young begin to fly, despite the fact that such recognition would appear to be useful... Moreover, we know that recognition is possible since young recognize their parents by voice shortly after hatching and parents do learn the calls of their young eventually" (Beecher

5 To be clear, I don't claim that we are innately set up to notice language-specific structures such as VERB or PASSIVE, only that we notice that there is language. 
1988:471). Among a number of possible avenues, natural selection can "increase perceptual sensitivity or attention to the signature traits" (Beecher 1988:467).

Differences in attention, alone or in conjunction with processing difficulties, may also be behind impaired language learning outcomes in children with autism spectrum disorder and delayed or abnormal language. Children with specific language impairment (SLI) have trouble processing language because of structural factors in the brain that distinguish them from non-impaired children. In contrast, autistic children with language impairment do not share these structural differences (Verhoeven et al. 2012; see, however, Lombardo et al. 2015), which suggests that processing may not be the language bottleneck for them. On the other hand, there is evidence that autistic children differ from their non-autistic peers in the way they allocate attention, especially to social, visual, or linguistic cues (Guillon et al. 2014; Brown \& Bebko 2012; Redcay et al. 2013; but see Dube et al. 2016). For instance, "children with autism are able to discriminate linguistic stimuli based on prosody but nevertheless show atypical attention to prosody in comparison to children with typical development" (Ploog, Banerjee \& Brooks 2009:754). Note that this is not an attentional gap regarding language, but rather seems to be "a positive capacity to treat more incoming stuff as signal and less as noise" (Clark 2016:225). The result of this is that there is an overwhelming amount of signal. This situation presents the possibility that a failure to learn language may not be the absolute lack of any ability to sense or process relevant inputs, but instead, a "gain" setting (Kanai et al. 2015) that is too low, relative to other inputs.

By no means is the foregoing conclusive evidence that what is selected for by evolution is an attentive specialization rather than a processing specialization, but it is suggestive. To put this in the framework of predictive processing, "evolution equips agents with (adaptive) prior beliefs that define what is surprising and enables momentary minimization of free energy" (Friston, Thornton \& Clark 2012:5). Specifically, I'm suggesting that humans have a prior belief that there is a signal in the verbalizations and gestures of other humans.

\subsection{Conclusion}

Linguists, philosophers, and psychologists have argued from analogy that the ability to echolocate must be a cognitive specialization, encoded genetically, and produced 
by natural selection for echolocation. The argument from analogy is far from their only argument, and defeating it will not disprove their claims. Nevertheless, it is one brick in their logical edifice, and I have shown that it has been misplaced.

The main problem is that those using the argument from analogy seem to have overlooked fact that humans have the latent ability to learn to echolocate with very high levels of proficiency. This fact invalidates the argument that a language faculty must be a cognitive specialization, encoded genetically, and produced by natural selection for language because echolocation is like that.

The argument for an innate, genetic, cognitive specialization for language in humans, from analogy to bat echolocation, then, does not hold. While such a situation is not precluded by the foregoing, other possibilities seem more reasonable conclusions. Specifically, it seems that the innate difference between humans and echolocating bats, when it comes to echolocation, may be in the innate propensity to expect and attend to the relevant input. It seems clear that echolocating bats instinctively attend to the fine details of the spatial information in sound, while humans rarely do. Analogously, humans naturally attend to phonology and syntax while other animals appear not to.

\section{Acknowledgements}

I very grateful to Lore Thaler for helpful suggestions. 


\section{References}

Aihara, Ikkyu, Emyo Fujioka \& Shizuko Hiryu. 2013. Qualitative and quantitative analyses of the echolocation strategies of bats on the basis of mathematical modelling and laboratory experiments. PLoS ONE 8(7). doi:10.1371/journal.pone.0068635.

Baker, C. L. 1979. Syntactic theory and the projection problem. Linguistic Inquiry 10(4). 533-581.

Beckner, Clay, Nick C. Ellis, Richard Blythe, John Holland, Joan Bybee, Jinyun Ke, Morten H. Christiansen, Diane Larsen-Freeman, William Croft \& Tom Schoenemann. 2009. Language is a complex adaptive system: Position paper. Language Learning 59(4). 1-26. doi:10.1111/j.1467-9922.2009.00533.x.

Beecher, Michael D. 1988. Kin recognition in birds. Behavior Genetics 18(4). 465482.

Bérzin, F. \& C. R. H. Fortinguerra. 1993. EMG study of the anterior, superior and posterior auricular muscles in man. Annals of Anatomy 175(2). 195-197. doi:10.1016/S0940-9602(11)80182-2.

Brown, S. M. \& J. M. Bebko. 2012. Generalization, overselectivity, and discrimination in the autism phenotype: A review. Research in Autism Spectrum Disorders 6(2). 733-740. doi:10.1016/j.rasd.2011.10.012.

Buchler, Edward R. 1980. The development of flight, foraging, and echolocation in the little brown bat (Myotis lucifugus). Behavioral Ecology and Sociobiology 6(3). 211-218. doi:10.1007/BF00569202.

Chater, Nick, Alexander Clark, John A. Goldsmith \& Amy Perfors. 2015. Empiricism and language learnability. Oxford: Oxford University Press. doi:10.1093/acprof:oso/9780198734260.

Clark, Andy. 2016. Surfing uncertainty: Prediction, action and the embodied mind. Oxford: Oxford University Press.

Culicover, Peter W. \& Ray Jackendoff. 2005. Simpler syntax. Oxford: Oxford University Press. doi:10.1093/acprof:oso/9780199271092.001.0001.

Dodsworth, C., L. J. Norman \& Lore. Thaler. 2020. Navigation and perception of spatial layout in virtual echo-acoustic space. Cognition 197(June 2019). Elsevier. 
104185. doi:10.1016/j.cognition.2020.104185.

Dube, William V., Rachel S. Farber, Marlana R. Mueller, Eileen Grant, Lucy Lorin \& Curtis K. Deutsch. 2016. Stimulus Overselectivity in Autism, Down Syndrome, and Typical Development. American Journal on Intellectual and Developmental Disabilities 121(3). 219-235. doi:10.1352/1944-7558-121.3.219.

Enattah, Nabil Sabri, Tine G. K. Jensen, Mette Nielsen, Rikke Lewinski, Mikko Kuokkanen, Heli Rasinpera, Hatem El-Shanti, et al. 2008. Independent introduction of two lactase-persistence alleles into human populations reflects different history of adaptation to milk culture. American Journal of Human Genetics 82(1). 57-72. doi:10.1016/j.ajhg.2007.09.012.

Evans, Nicholas \& Stephen C. Levinson. 2009. The myth of language universals: language diversity and its importance for cognitive science. The Behavioral and brain sciences 32(5). 429-448; discussion 448-494. doi:10.1017/S0140525X0999094X.

Fitch, W. Tecumseh. 2014. Toward a computational framework for cognitive biology: Unifying approaches from cognitive neuroscience and comparative cognition. Physics of Life Reviews 11(3). 329-364. doi:10.1016/j.plrev.2014.04.005.

Fitch, W. Tecumseh, Marc D. Hauser \& Noam Chomsky. 2005. The evolution of the language faculty: Clarifications and implications. Cognition 97. 179-210. doi:10.1016/j.cognition.2005.02.005.

Fortin, Madeleine, Patrice Voss, Catherine Lord, Maryse Lassonde, Jens Pruessner, Dave Saint-Amour, Constant Rainville \& Franco Lepore. 2008. Wayfinding in the blind: Larger hippocampal volume and supranormal spatial navigation. Brain 131. 2995-3005. doi:10.1093/brain/awn250.

Frisch, Karl von. 1967. The dance language and orientation of bees. Cambridge, Mass: The Belknap Press of Harvard University Press.

Friston, Karl J., Christopher Thornton \& Andy Clark. 2012. Free-energy minimization and the dark-room problem. Frontiers in Psychology 3(MAY). 1-7. doi:10.3389/fpsyg.2012.00130.

Gould, Edwin, Norman C. Negus \& Alvin Novick. 1964. Evidence for echolocation in shrews. Journal of Experimental Zoology 156(1). 19-37. 
Gould, Stephen J. \& Richard C. Lewontin. 1979. The spandrels of San Marco and the Panglossian paradigm: A critique of the adaptationist programme. Proceedings of the Royal Society of London. Series B 205. 581-198.

Gould, Stephen J. \& Elisabeth S. Vrba. 1982. Exaptation-A missing term in the science of form. Paleobiology 8(1). 4-15. doi:10.2307/2400563.

Griffin, Donald R. 1944. Echolocation by blind men, bats and radar. Science 100(2609). 589-590. doi:10.1126/science.100.2609.589.

Grinnell, Alan D. \& S. Hagiwara. 1972. Studies of auditory neurophysiology in nonecholocating bats, and adaptations for echolocation in one genus, Rousettus. Zeitschrift für Vergleichende Physiologie 76(1). 82-96. doi:10.1007/BF00395501.

Guillon, Quentin, Nouchine Hadjikhani, Sophie Baduel \& Bernadette Rog 2014. Visual social attention in autism spectrum disorder: Insights from eye tracking studies. Neuroscience and Biobehavioral Reviews 42. Elsevier Ltd. 279-297. doi:10.1016/j.neubiorev.2014.03.013.

Hare, Brian, Irene Plyusnina, Natalie Ignacio, Olesya Schepina, Anna Stepika, Richard Wrangham \& Lyudmila Trut. 2005. Social cognitive evolution in captive foxes is a correlated by-product of experimental domestication. Current Biology 15(3). 226-230. doi:10.1016/j.cub.2005.01.040.

Hauser, Marc D., Noam Chomsky \& W. Tecumseh Fitch. 2002. The faculty of language: what is it, who has it, and how did it evolve? Science (New York, N.Y.) 298(5598). 1569-1579. doi:10.1126/science.298.5598.1569.

Hauser, Marc D., Charles Yang, Robert C. Berwick, Ian Tattersall, Michael J. Ryan, Jeffrey Watumull, Noam Chomsky \& Richard C. Lewontin. 2014. The mystery of language evolution. Frontiers in Psychology 5(MAY). 1-12. doi:10.3389/fpsyg.2014.00401.

Heffner, R. S., G. Koay \& H. E. Heffner. 2006. Hearing in large (Eidolon helvum) and small (Cynopterus brachyotis) non-echolocating fruit bats. Hearing Research 221(1-2). 17-25. doi:10.1016/j.heares.2006.06.008.

Huddleston, Rodney \& Geoffrey K. Pullum. 2002. The Cambridge grammar of the English language. Cambridge: Cambridge Univ Press. 
Hurford, James. 2012. The origins of grammar: Language in the light of evolution II. Oxford: Oxford University Press.

Hutcheon, James M., John A. W. Kirsch \& Theodore Garland. 2002. A comparative analysis of brain size in relation to foraging ecology and phylogeny in the Chiroptera. Brain, behavior and evolution 60. 165-180. doi:10.1159/000065938.

Jackendoff, Ray. 2002. Foundations of Language. Oxford: Oxford University Press. doi:10.1215/00267929-4-1-96.

Jäger, Gerhard \& James Rogers. 2012. Formal language theory: Refining the Chomsky hierarchy. Philosophical Transactions of the Royal Society B: Biological Sciences 367. 1956-1970. doi:10.1098/rstb.2012.0077.

Jordan, Gabriele, Samir S. Deeb, Jenny M. Bosten \& J. D. Mollon. 2010. The dimensionality of color vision in carriers of anomalous trichromacy. Journal of vision 10(8). 12. doi:10.1167/10.8.12.

Kanai, Ryota, Yutaka Komura, Stewart Shipp, Karl J. Friston, Yutaka Komura \& Stewart Shipp. 2015. Cerebral hierarchies: predictive processing, precision and the pulvinar. Philosophical transactions of the Royal Society of London. Series B, Biological sciences 370. 20140169. doi:10.1098/rstb.2014.0169.

Kolarik, Andrew J., Silvia Cirstea, Shahina Pardhan \& Brian C. J. Moore. 2014. A summary of research investigating echolocation abilities of blind and sighted humans. Hearing Research 310. Elsevier B.V. 60-68. doi:10.1016/j.heares.2014.01.010.

Liberman, Alvin M., Franklin. S. Cooper, Donald. P. Shankweiler \& Michael Studdert-Kennedy. 1967. Perception of the speech code. Psychological Review 74(6). 431-461.

Lombardo, Michael V., Karen Pierce, Lisa T. Eyler, Cindy CarterBarnes, Clelia Ahrens-Barbeau, Stephanie Solso, Kathleen Campbell \& Eric Courchesne. 2015. Different functional neural substrates for good and poor language outcome in autism. Neuron 86(2). 567-577. doi:10.1016/j.neuron.2015.03.023.

Lord, Kathryn A., Greger Larson, Raymond P. Coppinger \& Elinor K. Karlsson. 2020. The History of Farm Foxes Undermines the Animal Domestication Syndrome. Trends in Ecology and Evolution 35(2). 125-136. 
doi:10.1016/j.tree.2019.10.011.

Maguire, Eleanor A., David G. Gadian, Ingrid S. Johnsrude, Catriona D. Good, John Ashburner, Richard S. J. Frackowiak \& Christopher D. Frith. 2000. Navigationrelated structural change in the hippocampi of taxi drivers. Proceedings of the National Academy of Sciences of the United States of America 97(8). 4398-4403. doi:10.1073/pnas.070039597.

McGrath, Richard, Thomas Waldmann \& Mikael Fernström. 1999. Listening to rooms and objects. AES 16th International Conference on Spatial Sound Reproduction. 1-11. http://www.aes.org/e-lib/browse.cfm?elib=8008.

Pepperberg, Irene. M. 1983. Cognition in the African Grey parrot: Preliminary evidence for auditory/vocal comprehension of the class concept. Animal Learning \& Behavior 11(2). 179-185. doi:10.3758/BF03199646.

Pinker, Steven \& Paul Bloom. 1990. Natural language and natural selection. Behavioral and Brain Sciences 13. 707-784.

Pinker, Steven \& Ray Jackendoff. 2005. The faculty of language: What's special about it? Cognition 95. 201-236. doi:10.1016/j.cognition.2004.08.004.

Ploog, Bertram O., Snigdha Banerjee \& Patricia J. Brooks. 2009. Attention to prosody (intonation) and content in children with autism and in typical children using spoken sentences in a computer game. Research in Autism Spectrum Disorders 3(3). 743-758. doi:10.1016/j.rasd.2009.02.004.

Polat, Uri, Clifton Schor, Jian-Liang Tong, Ativ Zomet, Maria Lev, Oren Yehezkel, Anna Sterkin \& Dennis M. Levi. 2012. Training the brain to overcome the effect of aging on the human eye. Scientific Reports 2. 2-7. doi:10.1038/srep00278.

Pullum, Geoffrey K. \& Barbara C. Scholz. 2002. Empirical assessment of stimulus poverty arguments. The Linguistic Review 19(i). 9-50.

Putnam, Hilary. 1992. What is innate and why: comments on the debate. The Philosophy of mind: classical problems/contemporary issues. 397.

Rademacher, J., P. Morosan, T. Schormann, A. Schleicher, C. Werner, H. J. Freund \& K. Zilles. 2001. Probabilistic mapping and volume measurement of human primary auditory cortex. NeuroImage 13. 669-683.

doi:10.1006/nimg.2000.0714. 
Redcay, Elizabeth, David Dodell-Feder, Penelope L. Mavros, Mario Kleiner, Mark J. Pearrow, Christina Triantafyllou, John D. Gabrieli \& Rebecca Saxe. 2013. Atypical brain activation patterns during a face-to-face joint attention game in adults with autism spectrum disorder. Human Brain Mapping 34(10). 25112523. doi:10.1002/hbm.22086.

Roberts, Laurence H. 1979. Correlation of respiration and ultrasound production in rodents and bats. Journal of Zoology 168. 439-449. doi:10.1111/j.14697998.1972.tb01360.x.

Rombaux, Philippe, Caroline Huart, Anne G. De Volder, Isabel Cuevas, Laurent Renier, Thierry Duprez \& Cecile Grandin. 2010. Increased olfactory bulb volume and olfactory function in early blind subjects. Neuroreport 21. 10691073. doi:10.1097/WNR.0b013e32833fcb8a.

Rosenblum, Lawrence D., Michael S. Gordon \& Luis Jarquin. 2000. Echolocating distance by moving and stationary listeners. Ecological Psychology 12(3). 181206. doi:10.1207/S15326969ECO1203_1.

Rosenzweig, Mark R., Donald A. Riley \& David Krech. 1955. Evidence for echolocation in the rat. Science 121(3147). 600. doi:10.1126/science.121.3147.600.

Schenkman, Bo N. \& Mats E. Nilsson. 2010. Human echolocation: Blind and sighted persons' ability to detect sounds recorded in the presence of a reflecting object. Perception 39. 483-501. doi:10.1068/p6473.

Schuller, Gerd \& George Pollak. 1979. Disproportionate frequency representation in the inferior colliculus of doppler-compensating Greater Horseshoe bats: Evidence for an acoustic fovea. Journal of Comparative Physiology 132(1). 4754. doi:10.1007/BF00617731.

Seligman, Martin E. P. 2016. Phobias and preparedness - Republished article. Behavior Therapy 47(5). 577-584. doi:10.1016/j.beth.2016.08.006.

Shibasaki, Masahiro \& Nobuyuki Kawai. 2009. Rapid detection of snakes by Japanese monkeys (Macaca fuscata): an evolutionarily predisposed visual system. Journal of comparative psychology 123(2). 131-135. doi:10.1037/a0015095. 
Siemers, Björn M, Grit Schauermann, Hendrik Turni \& Sophie von Merten. 2009. Why do shrews twitter? Communication or simple echo-based orientation. Biology letters 5(5). 593-596. doi:10.1098/rsb1.2009.0378.

Slobodchikoff, C. N., Andrea Paseka \& Jennifer L. Verdolin. 2009. Prairie dog alarm calls encode labels about predator colors. Animal Cognition 12(3). 435-439. doi:10.1007/s10071-008-0203-y.

Speakman, John R. 1993. The evolution of echolocation for predation. Symposia of the Zoological Society of London 65. 39-63.

Stroffregen, Thomas A. \& John B. Pittenger. 1995. Human echolocation as a basic form of perception and action. Ecological Psychology 7(2015). 181-216. doi:10.1207/s15326969eco0703_2.

Teng, Santani, Amrita Puri \& David Whitney. 2012. Ultrafine spatial acuity of blind expert human echolocators. Experimental Brain Research 216. 483-488. doi:10.1007/s00221-011-2951-1.

Teng, Santani \& David Whitney. 2011. The acuity of echolocation: Spatial resolution in the sighted compared to expert performance. Journal of Visual Impairment and Blindness 105(1). 20-32.

Thaler, Lore. 2013. Echolocation may have real-life advantages for blind people: An analysis of survey data. Frontiers in Physiology 4. 1-8. doi:10.3389/fphys.2013.00098.

Thaler, Lore, Stephen R. Arnott \& Melvyn A. Goodale. 2011. Neural correlates of natural human echolocation in early and late blind echolocation experts. PLoS ONE 6(5). doi:10.1371/journal.pone.0020162.

Tooby, John, Leda Cosmides \& H. Clark Barrett. 2005. Resolving the debate on innate ideas. In Peter Carruthers, Stephen Laurence \& Stephen Stich (eds.), The innate mind: Structure and contents, 305-337. Oxford: Oxford University Press.

Verhoeven, Judith S., Nathalie Rommel, Elena Prodi, Alexander Leemans, Inge Zink, Ellen Vandewalle, Ilse Noens, et al. 2012. Is there a common neuroanatomical substrate of language deficit between autism spectrum disorder and specific language impairment? Cerebral Cortex 22(10). 2263-2271. doi:10.1093/cercor/bhr292. 
Vernes, Sonja C. 2017. What bats have to say about speech and language.

Psychonomic Bulletin and Review 24(1). Psychonomic Bulletin \& Review. 111117. doi:10.3758/s13423-016-1060-3.

Weinbeer, Moritz \& Elisabeth K. V. Kalko. 2007. Ecological niche and phylogeny: The highly complex echolocation behavior of the trawling long-legged bat, Macrophyllum macrophyllum. Behavioral Ecology and Sociobiology 61(9). 1337-1348. doi:10.1007/s00265-007-0364-8.

Whitney, Glayde \& David B. Harder. 1994. Genetics of bitter perception in mice. Physiology and Behavior 56(6). 1141-1147. doi:10.1016/0031-9384(94)903581.

Wilkinson, Gerald S. 2003. Social and vocal complexity in bats. Animal social complexity, 322-341. Harvard University Press. doi:10.4159/harvard.9780674419131.c24.

Yovel, Yossi, Maya Geva-Sagiv \& Nachum Ulanovsky. 2011. Click-based echolocation in bats: Not so primitive after all. Journal of Comparative Physiology A: Neuroethology, Sensory, Neural, and Behavioral Physiology 197(5). 515-530. doi:10.1007/s00359-011-0639-4.

Zhou, Yifeng, Changbing Huang, Pengjing Xu, Liming Tao, Zhuping Qiu, Xiangrui Li \& Zhong Lin Lu. 2006. Perceptual learning improves contrast sensitivity and visual acuity in adults with anisometropic amblyopia. Vision Research 46(5). 739-750. doi:10.1016/j.visres.2005.07.031. 